Veritas Et Scientia

Vol. $8, \mathrm{~N}^{\circ} 2$.

Julio - diciembre del 2019.

ISSN Edición Online: 2617-0639

https://doi.org/10.47796/ves.v8i2.137

\title{
COMPETENCIAS LABORALES PARA LA GESTIÓN PÚBLICA Y PRODUCTIVIDAD DE LAS UNIDADES ORGÁNICAS DE UN MUNICIPIO PROVINCIAL.
}

\section{LABOR COMPETENCES FOR PUBLIC MANAGEMENT AND PRODUCTIVITY OF ORGANIC UNITS OF A PROVINCIAL MUNICIPALITY}

\author{
Abigail Noemi Vicente Herrera ${ }^{1}$ \\ Elena Miriam Chávez Garcés²
}

Aceptado: $17 / 09 / 2019$

Publicado online:15/01/2020

\begin{abstract}
RESUMEN
El objetivo de esta investigación es explicar de qué manera las competencias laborales para la gestión pública afecta en la productividad de las unidades orgánicas de la Municipalidad Provincial Jorge Basadre, en el año 2018. Es una investigación de tipo básica - cuantitativa, no experimental, explicativa. En la presente investigación, se ha tomado información de 108 trabajadores, del total de la población; se utilizó como instrumento un test de competencias laborales y una ficha de registro documental para consignar los datos de la productividad. La validación de contenido fue mediante la opinión de expertos, la prueba de confiabilidad del test se realizó mediante el método Alfa de Cronbach, habiéndose obtenido un indicador alfa de 0.866 puntos. Los resultados de las competencias laborales, reportan una media de 44.54, según la escala se encuentra en el nivel regular, la medición de los indicadores reporta que $63.9 \%$ de los evaluados tiene nivel regular de conocimientos, $59.3 \%$ con habilidades regulares y $67.6 \%$ con actitudes indiferentes. Los resultados de la productividad, indican una puntuación de 49.14 que significa un nivel de avance en proceso, el análisis de las actividades de cada unidad orgánica reporta que la Sub Gerencia de Liquidación de Proyectos posee el menor promedio con un $26.67 \%$ y la Sub Gerencia de Contabilidad posee el mayor promedio con un $70.0 \%$. Existe correlación positiva alta, según coeficiente de correlación $r$, entre las competencias laborales y la
\end{abstract}

Maestro en Gestión y Políticas Públicas. Ingeniera Ambiental Colegiada, Especialista en Gestión de la Calidad y Auditoría Ambiental, experiencia laboral como Docente Universitaria en la Escuela Profesional de Ingeniería Ambiental de la Universidad Privada de Tacna, Tacna, Perú

2 Maestro en Ciencias, Implantador MEF Ministerio de Economía y Finanzas, Tacna, Perú 
productividad de las unidades orgánicas de la Municipalidad Provincial de Jorge Basadre.

Palabras claves: Competencias laborales, productividad y unidades orgánicas.

\section{ABSTRACT}

The objective of this research is to explain how labor competencies for public management affect the productivity of the organic units of the Jorge Basadre Provincial Municipality, in 2018. It is a basic type research - quantitative, not experimental, explanatory. In the present investigation, information has been taken from 108 workers, of the total population; A work skills test and a documentary record form were used as an instrument to record productivity data. The content validation was by expert opinion, the test of reliability of the test was performed using the Cronbach's Alpha method, having obtained an alpha indicator of 0.866 points. The results of the labor competencies, report an average of 44.54, according to the scale is in the regular level, the measurement of the indicators reports that $63.9 \%$ of those evaluated have a regular level of knowledge, $59.3 \%$ with regular skills and $67.6 \%$ with indifferent attitudes. The productivity results indicate a score of 49.14 which means a level of progress in progress, the analysis of the activities of each organic unit reports that the Project Liquidation Sub-Division has the lowest average with $26.67 \%$ and the Sub-Management Accounting has the highest average with $70.0 \%$. The hypothesis test with Pearson's correlation coefficient $r$ gives us the sig. Bilateral $=0.029<0.05$, therefore, the null hypothesis is rejected and the alternate hypothesis is accepted. In conclusion, if there is a significant effect of labor competencies on the productivity of the organic units of the Provincial Municipality of Jorge Basadre, in this way, confirming that there is a high positive correlation between labor competencies and productivity in the Provincial Municipality Jorge Basadre.

Keywords: Labor competencies, productivity and organic units.

\section{INTRODUCCIÓN}

En la actualidad las organizaciones se vuelven más competitivas, donde la capacidad de los trabajadores se vuelve más vulnerable en cuanto a su desempeño, en este sentido el incremento de la productividad se convierte en un principal objetivo, por ende las entidades públicas toman mayor importancia al servicio que se ofrece a la población; sin embargo, se olvidan de analizar las dificultades que tiene el personal que labora en ella, observando que existen carencias respecto a las competencias laborales, puesto que no poseen los conocimientos, habilidades y actitudes suficientes que requiere el puesto en que se desempeñan laboralmente. Tal situación genera deficiencias en la productividad, específicamente en el logro de las metas programadas por las unidades orgánicas. Bajo este enfoque el objeto de la presente investigación es explicar de qué manera las competencias laborales para la gestión pública afecta a la productividad de las unidades orgánicas de la Municipalidad Provincial Jorge Basadre, en el año 2018. En el Perú las entidades públicas tienen como razón principal prestar servicios a una comunidad, estas 
se caracterizan por las diversas actividades que realizan a fin de lograr satisfacer las necesidades de la población. En ese proceso se requiere que el personal se encuentre capacitado, y tenga las competencias laborales necesarias para desempeñar de forma eficiente las labores asignadas en su puesto de trabajo como parte de la estructura funcional de la entidad pública. Según el diario El Peruano (2018), un estudio realizado por la Universidad del Pacífico a 1,500 servidores públicos, reveló que solo uno de cada cinco había completado sus estudios de post grado. Es decir, un $82 \%$ no cuenta con maestrías ni doctorados en su trayectoria. Un servidor público debidamente capacitado es un gestor con mayor competencia para cumplir su función, y al mismo tiempo un representante del Estado que contribuirá a beneficiar al ciudadano con servicios de calidad. El tema de la calidad basada en la eficiencia siempre vuelve a debate en torno a procesos de modernización del Estado, pues se revela como una importante alternativa para la mejora continua y la búsqueda de legitimidad de los gobiernos o instituciones públicas. Condori (2013), concluyó que existe una influencia significativa a las competencias laborales que caracterizan al personal sobre su desempeño cuyo factor determinante de la problemática es la alta rotación del personal. Facho Gutiérrez (2017) refiere que es necesario aplicar programas para mejorar el rendimiento laboral. Sánchez (2012), concluye que en las últimas décadas se ha denotado una gran evolución de los procesos orientados a la formación de las personas que buscan como propósito fundamental contribuir con la profesionalización y además con el crecimiento de las organizaciones e instituciones. El nivel de tenencia para la productividad tiende a reducirse si los trabajadores no reciben capacitación y actualización (Perea Guerra,2014). Los niveles de productividad siempre pueden mejorar si es que existe objetivos claros conocidos por los trabajadores (Vásquez Casas, 2017.

Martínez (2013), precisó algunas características que se deben tener en cuenta para calificar las competencias citando a CONOCER (2014) las cuales: son aprendidas, conscientes, autónomas, permanentes, complejas, contextos variados, generan resultados e implican eficiencia. Indicadores de la Competencia Laboral. Un individuo puede incluir su comportamiento a las competencias (Cantera Herrero,1995). Así mismo según Spencer y Spencer plantea el modelo del iceberg y explica que las competencias se dividen en dos grupos que son: las fáciles de detectar y desarrollar, como: las habilidades o destrezas y conocimientos; y las difíciles de detectar y desarrollar, como: el concepto de uno mismo, las actitudes, valores, y el núcleo mismo de la personalidad. Así también Ontoria, A.; Gómez R.; Molina, A. (2002), establecen que "Existen diferencias entre información y conocimientos; aquella se compone de datos y acontecimientos, mientras que el conocimiento se relaciona con la comprensión y el significado que se da a la información." No basta solo saber la técnica, sino que se debe saber aplicar en la realidad. (Capella, 1999). Además, según Miguel, (1991) una "Organización es los sentimientos de las creencias y los valores, así como predisposición de una persona para comportarse de una manera dada". La productividad laboral también se puede medir en función del tiempo, esto quiere decir que mientras menos tiempo se emplee en cumplir con una meta programada, podemos considerar que entidad pública es más productiva, así mismo Ramirez Cavassa (2010) define "La productividad es la relación entre insumos y productos, en este sentido se considera como insumos el trabajo, es el total de las horas trabajadas". Asimismo, Chiavenato 
(2007), menciona: La productividad se considera el efecto (y no causa) de la administración de varios recursos. La productividad es la relación mensurable que existe entre el producto obtenido (resultado o salida) y los recursos empleados para su producción. Según Gomes, (2008), "la productividad es la medida del valor que añade el empleado particular a los bienes o servicios que produce la organización. Cuanto mayor es la producción por individuo, mayor será la productividad de la organización. En las entidades públicas también se realiza la medición de la productividad, demostrando que se está utilizando de manera adecuada todos los recursos asignados tales como recursos humanos, mobiliario y tecnologías, los que permiten alcanzar objetivos y metas programadas, atendiendo la demanda social y ofreciendo un servicio de calidad a la población.

\section{OBJETIVOS}

a. Identificar el nivel de competencias laborales para la gestión pública en las unidades orgánicas de la Municipalidad Provincial Jorge Basadre, en el año 2018.

b. Conocer el nivel de productividad en las unidades orgánicas de la Municipalidad Provincial Jorge Basadre, en el año 2018.

\section{METODOLOGÍA}

Investigación de tipo básica, no experimental, de nivel explicativo. El ámbito de la investigación se limita a conocimientos de la gestión de recursos humanos que participan en los procesos de la gestión pública, el cual, se realizará en la Municipalidad Provincial Jorge Basadre, del departamento de Tacna, Región Tacna. El tiempo de investigación corresponde al año 2018. La Población está constituida por un promedio de 108 trabajadores municipales que comprende de gerentes, jefes y personal administrativo de la Municipalidad. Se utilizó como instrumento un test de competencias sobre los conocimientos, habilidades y actitudes. La validación de la ficha de registro documental se sometió a la validez de contenido mediante el método de opinión de expertos, y la validación del test de competencias laborales se sometió a la prueba de validez de confiabilidad mediante el método Alfa de Cronbach con un alfa de $86.6 \%$ de fiabilidad.

\section{RESULTADOS}

El nivel de conocimientos del personal en gestión pública, es bajo en un $2.8 \%$, el $63.9 \%$ regular y el $33.3 \%$ tiene un nivel de conocimientos medio (Tabla 01). La tabla 2 presenta información relacionada nivel de habilidades para la gestión pública, donde apreciamos que el $6.5 \%$ es bajo, el $59.3 \%$ regular y el $34.3 \%$ señala el nivel de las habilidades que posee el personal es medio. La tabla 3 presenta información relacionada el nivel de actitudes para la gestión pública, donde apreciamos que el $4.6 \%$ es negativa, el $67.6 \%$ indiferente y el $27.8 \%$ actitudes positivas. 
Tabla 1

Nivel de conocimientos sobre la gestión pública

\begin{tabular}{lcc}
\hline & $\mathbf{n}$ & $\mathbf{\%}$ \\
\hline Bajo & 3 & 2,8 \\
Regular & 69 & 63,9 \\
Alto & 36 & 33,3 \\
\hline Total & 108 & 100,0 \\
\hline
\end{tabular}

Tabla 2

Nivel de habilidades para la gestión pública

\begin{tabular}{lrr}
\hline & $\mathbf{n}$ & $\%$ \\
\hline Bajo & 7 & 6,5 \\
Regular & 64 & 59,3 \\
Alto & 37 & 34,3 \\
Total & 108 & 100,0 \\
\hline
\end{tabular}

Tabla 3

Nivel de actitudes para la gestión pública

\begin{tabular}{lcc}
\hline & $\mathbf{n}$ & $\mathbf{\%}$ \\
\hline Negativa & 5 & 4,6 \\
Indiferente & 73 & 67,6 \\
Positiva & 30 & 27,8 \\
Total & 108 & 100,0 \\
\hline
\end{tabular}

De la información anterior deducimos que la dimensión con mayor desempeño son las actitudes que posee el personal. Sin embargo, no representa un nivel óptimo, mientras que los conocimientos y las habilidades representan niveles bajos para la gestión pública.

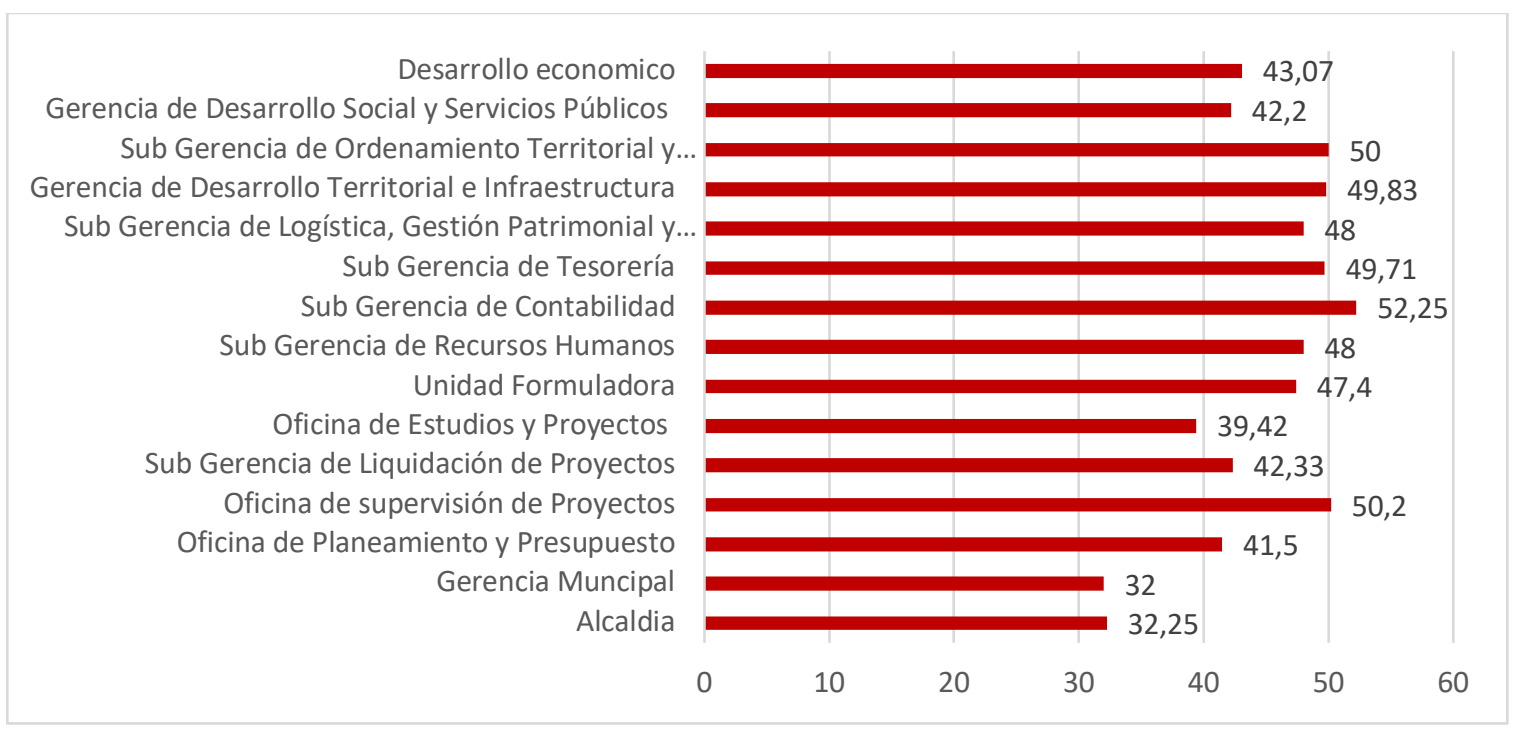

Figura 1

Nivel de competencias laborales según unidad orgánica de la MPJB 


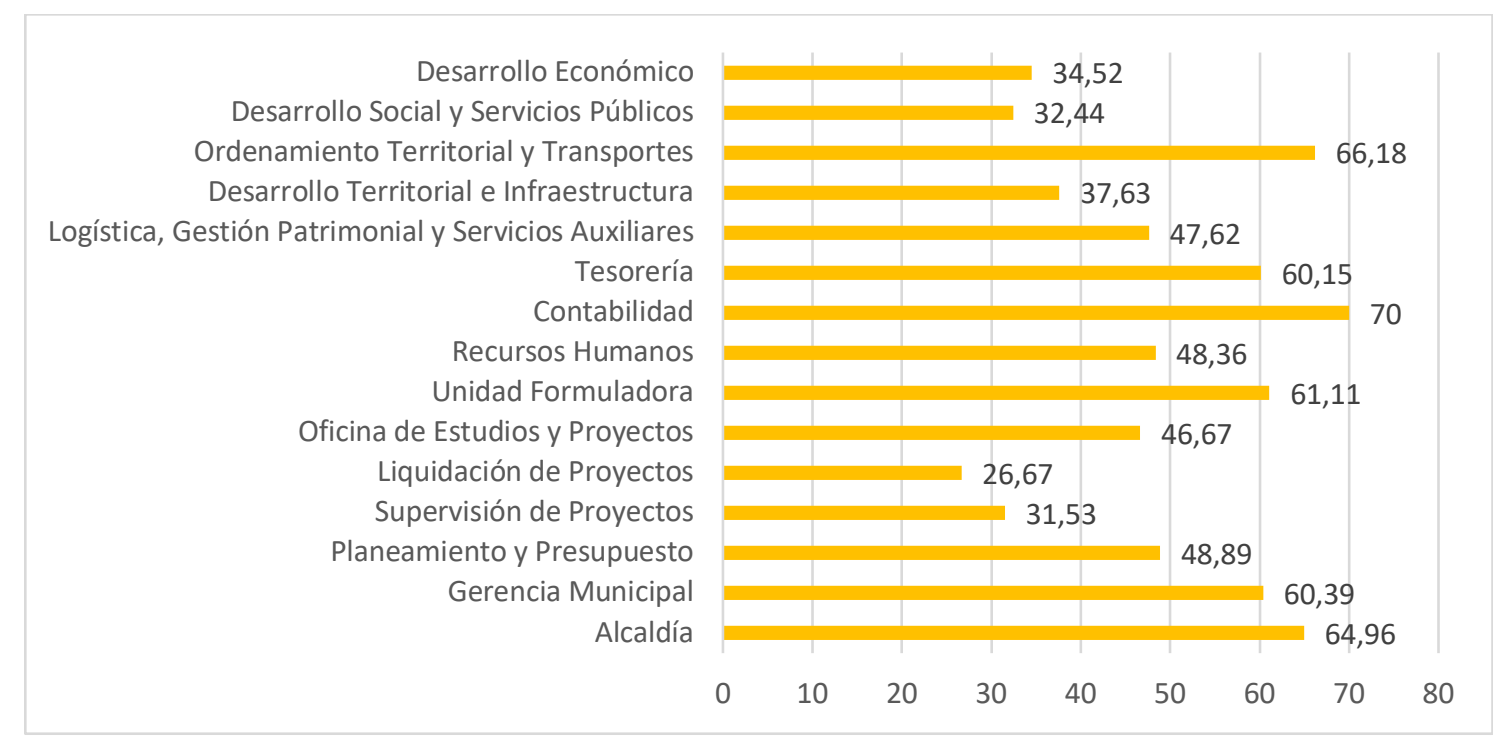

Figura 1

Productividad por unidades orgánicas de la MPJB

\begin{tabular}{llrr}
\hline & \multicolumn{2}{c}{ Correlaciones } & \\
\hline \multirow{3}{*}{ Productividad } & Productividad & Competencias \\
\hline & Correlación de Pearson & 1 & 0,753 \\
\cline { 2 - 4 } & Sig. (bilateral) & 15 & 0,029 \\
& $\mathrm{~N}$ & 0,753 & 15 \\
\hline \multirow{3}{*}{ Competencias } & Correlación de Pearson & 0,029 & 1 \\
\cline { 2 - 4 } & Sig. (bilateral) & 15 & 15 \\
& $\mathrm{~N}$ & & \\
\hline
\end{tabular}

Los resultados muestran que existe correlación positiva alta entre las competencias laborales y la productividad en la Municipalidad Provincial Jorge Basadre $(r=0.753)$.

\section{DISCUSIÓN}

En relación a la productividad de las unidades orgánicas de la Municipalidad Provincial Jorge Basadre; apreciamos una media de 49.14 que significa un nivel de avance de metas que están en proceso, de la información anterior se puede observar una Varianza de 201,14; Desviación estándar 14.18 es el grado de dispersión que se encuentran los promedios de productividad de cada unidad orgánica; al respecto en la investigación realizada por Facho Gutiérrez (2017), estudia la Productividad Laboral en Trabajadores de la Subgerencia de Operaciones de Fiscalización, Municipalidad de Lima, en la Tabla 4 de manera general indican que hay 42 empleados es decir $73.7 \%$ que califican la gestión administrativa como regular y tienen una productividad laboral regular lo cual muestra una posible relación entre las variables mencionadas.

Existe un efecto de las competencias laborales en la productividad de las unidades orgánicas, por tanto, siendo las dimensiones más destacadas las habilidades y actitudes, en cuanto a los conocimientos sobre gestión pública, se tiene que mejorar 
principalmente en la capacitación en temas de gestión pública y procedimientos inherentes de cada unidad orgánica en la Municipalidad Provincial Jorge Basadre. La productividad de las unidades orgánicas de la Municipalidad Provincial Jorge Basadre está en proceso. Se recomienda que la Alta Dirección de la Municipalidad Provincial Jorge Basadre, realice acciones de monitoreo para contribuir al mejoramiento del nivel de competencias laborales, priorizando los conocimientos sobre gestión pública, habilidades y actitudes necesarias para lograr el cumplimiento de las metas institucionales, ello permitirá mejorar los niveles de Productividad de cada unidad orgánica de las unidades orgánicas de la Municipalidad Provincial Jorge Basadre. Para mejorar el nivel de competencias laborales, se recomienda a la Sub Gerencia de Recursos Humanos priorice la evaluación del perfil de cada profesional en el proceso de contratación de personal, específicamente en los aspectos de conocimientos y capacitaciones sobre la gestión pública. Así como también ejecutar un programa de mejoramiento continuo que conduzca a la optimización de procesos, capacitaciones del personal para mejorar su desarrollo personal y el desempeño de sus funciones en relación al cargo que ocupa. Para mejorar el nivel de productividad de las unidades orgánicas de la Municipalidad Provincial Jorge Basadre, la Gerencia Municipal debe realizar evaluaciones periódicas en relación al desarrollo de las actividades programadas por cada unidad orgánica, con el propósito de determinar el cumplimiento de las metas institucionales; y como consecuencia de ellos implementar medidas preventivas y efectivas para mejorar la gestión.

\section{REFERENCIAS BIBLIOGRÁFICAS}

Alles, M. (2005). Gestión por Competencias, El Diccionario, 2da.Edición. Buenos Aires- Argentina: Editorial Granica.

Atanasof, A. N. (2018). Competencias laborales en la Administración Pública. Obtenido de Competencias laborales en la Administración Pública: Bazinet, A. (1984). La Evaluación del rendimiento: métodos para la evaluación de los mandos intermedios en la empresa. Barcelona: Editorial Herder.

Becerra Gálvez, Mayeline; Campos Ahumada, Francisca. (2012). El Enfoque por Competencias y sus Aportes en la Gestión de Recursos Humanos. Obtenido de Bunk, G. P. (1994). La transmisión de las competencias en la formación y perfeccionamiento profesionales en la RFA. Bélgica: Revista Cedefop N ${ }^{\circ} 1$. Editorial Diamantidi.

Capella J.; \& Sánchez Moreno G. (1999). Aprendizaje y constructivismo. Lima: Ediciones Massey and Vanier.

Chiavenato, I. (2007). Administración de Recursos Humanos. Santa Fé de Bogotá: Colombia: Mc Graw Hill.

Condori Ramos, G. M. (2011). Las competencias laborales y su influencia en el desempeño del personal de la Municipalidad distrital Coronel Gregorio Albarracín Lanchipa de Tacna, en el año 2011 (Tesis). Tacna.

Condori Ramos, G. M. (2013). Las Competencias Laborales y Su Influencia en el Desempeño del Personal de la Municipalidad Distrital y Coronel Gregorio Albarracín Lanchipa de Tacna, en el año 2011 - Tesis. TACNA.

CONOCER Conocimiento, C. y. (2014). Desarrollo de Estándares de Competencia (EC) y Diseño de Instrumentos de Evaluación de Competencia - Versión 7.0 - Mexico. Obtenido de Consejo de Normalización y Certificación de Competencia Laboral: Cuba, T. (2000). Introducción a la psicología. Lima: Universidad Inca Garcilaso de la Vega. 
El Peruano, D. (4 de Julio de 2013). LEY №30057 DEL SERVICIO CIVIL. Lima, Perú.

El Peruano, D. (2018). "Capacitación y Desafíos de la Gestión Pública". Obtenido de El Peruano, D. (19 de Diciembre de 2018). DECRETO SUPREMO № 123-2018-PCM. Aprueba el Reglamento del Sistema Administrativo de Modernización de la Gestión Pública. Lima, Perú.

Ernst \& Young, C. (2008). Manual del Director de Recursos Humanos. Obtenido de Gestión por Competencias:. Obtenido de Manual del Director de Recursos Humanos.

Facho Gutiérrez, J. E. (2017). "Gestión Administrativa y Productividad Laboral en Trabajadores de la Subgerencia de Operaciones de Fiscalización, Municipalidad de Lima, 2016" (Tesis).

Fernández, G. (2004). Las Competencias. En G. Fernández, Las Competencias: Clave para una Gestión Integrada de los Recursos Humanos (pág. 186). España: Ediciones Deusto.

Frisancho, A. (2012). Bases biológicas y psicológicas del comportamiento humano. Lima: Universidad Nacional Mayor de San Marcos.

García, J. (2011). La Gestión de RRHH por competencias. Obtenido de Gomes, B. C. (2008). Gestion de Recursos Humanos (5a Edicion). En B. C. Gomes, Gestion de Recursos Humanos (5 $\underline{a}$ Edicion). Madrid, España: Pearson Educacion.

Hellriegel, D. Jackson, S. y Slocum, J. . (2002). Administración: Un Enfoque Basado en Competencias. 9na. Edición. México: Edicitorial: Thomson Editores, S.A. de C.V.

Hernández, R., Fernández, C. \& Batista P. . (2010). Fundamentos de metodología de la investigación. México: Editorial: Mc Graw Hill.

Institucional, P. -P. (2017). Municipalidad Provincial Jorge Basadre. Obtenido de Koontz, H. y Weirhrich, H. . (2004). Administración: Una vision global. México: Editorial McGraw Hill Interamericana.

Lomas Púa, R. (2017). Satisfacción laboral y su relación con la productividad de los trabajadores de la Municipalidad Distrital de Buenos Aires, en el año 2017 (Tesis). Tarapoto-Perú.

María del Carmen Ortego Maté; Santiago López González; María Lourdes Álvarez Trigueros. (s.f.). Ciencias Psicosociales I - UC. Obtenido de Temas 4. Las Actitudes: Martines, V. (1998). Productividad Laboral.

Martínez, V. (2013). Gestión del Talento Humano por competencias para una empresa de las Artes Gráficas. (Tesis, México: Instituto Politécnico Nacional de México.). Obtenido de Mucci, O. O. (2007). Las competencias laborales - Facultad de Ciencias Económicas y Sociales - Unmdp. Ontoria, A.; Gómez R.; Molina, A. . (2002). Potenciar la capacidad de aprender y pensar. Madrid: Narcea S.A.

Perea Guerra, N. P. (2014). "Estudio de las competencias de los recursos humanos en las buenas prácticas del sistema de administración financiera en las municipalidades distritales de Soplín y Capelo de la provincia de Requena, año 2014" (Tesis). Rábago, E. (2010). Gestión por competencias. Un enfoque para mejorar el rendimiento personal y empresarial. España.

Ramirez Cavassa, C. (2010). Ergonomia y Productividad. En C. R. Cavassa, Ergonomia y Productividad (pág. 253). México: Editorial Limusa.

Sánchez, E. (2012). Las Competencias Laborales En el Desempeño de la Función delContador Publico en las Fuerzas Armadas Nacionales: Clave en el Éxito Institucional (Tesis).

Schroeder, R. G. (2002). Administración de operaciones. McGraw Hill.

Spencer, S. \&. (1993). Diccionario de competencias. New York: John Wiley and Sons.

Vander, E. (2005). Gestión y Gerencias Empresariales. Eco Ediciones.

Vásquez Casas, J. G. (2017). El Clima Laboral y su Influencia en la Productividad de los Trabajadores Administrativos de la Municipalidad Distrital De Ciudad Eten, 2016 (Tesis). 